\title{
On Bifurcations in Nonlinear Consensus Networks
}

\author{
Vaibhav Srivastava \\ Jeff Moehlis \\ Francesco Bullo
}

\begin{abstract}
The theory of consensus dynamics is widely employed to study various linear behaviors in networked control systems. Moreover, nonlinear phenomena have been observed in animal groups, power networks and in other networked systems. This inspires the development in this paper of two novel approaches to define distributed nonlinear dynamical interactions. The resulting dynamical systems are akin to higher-order nonlinear consensus systems. Over connected undirected graphs, the resulting dynamical systems exhibit various interesting behaviors that we rigorously characterize.
\end{abstract}

\section{INTRODUCTION}

Collective behavior in animal groups, such as schools of fish, flocks of birds, and herds of wildebeests, is a widely studied phenomenon. It has been proposed that the decision making in such groups is distributed rather than central: each individual in such a group decides how to behave based on local information. In particular, some adjacency-based averaging models have been proposed to model the observed behavior in such systems. These adjacency-based averaging algorithms are called consensus algorithms, and have been widely studied in various engineering applications.

Of particular interest are recent results in ecology [4] which show that, for small difference in the preferences of the individuals, the decision making in animal groups is well modeled using consensus dynamics, but for significant differences in the preferences of individuals, the decision dynamics bifurcate away from consensus. This provides motivation for coming up with dynamics which mimic such nonlinear behaviors in engineered multi-agent systems.

Recently, dynamical systems theory has been extensively applied to networked systems. In particular, the consensus problem has been studied in various fields, e.g., network synchronization [15], flocking [18], rendezvous [10], sensor fusion [16], formation control [5], etc; a detailed description is presented in [12], [6]. Some nonlinear phenomena have been studied in certain classes of networks. Certain nonlinear protocols to achieve consensus have been studied [1]. The bifurcation problem has been studied in neural networks; a Hopf-like bifurcation has been observed in a two cell autonomous system [20], and pitchfork and Hopf bifurcations have been studied in artificial neural networks [14], [19]. Some static bifurcations have been studied in load flow dynamics of power networks [9]. A version of bifurcations in consensus networks has been studied in the opinion dynamics literature [11]. The models in opinion dynamics problems can be interpreted as consensus dynamics on a time varying

This work was supported in part by AFOSR MURI grant FA9550-07-10528.

Vaibhav Srivastava, Jeff Moehlis and Francesco Bullo are with the Department of Mechanical Engineering, University of California, Santa Barbara, Santa Barbara, CA 93106, USA, \{vaibhav, moehlis, bullo\}@engineering.ucsb.edu graph with no globally reachable node. These models are complicated, and are difficult to implement on an engineered multi-agent network.

In this paper, we propose distributed algorithms to achieve nonlinear behaviors in a networked system. We define two frameworks, namely, the absolute nonlinear flow, and the disagreement nonlinear flow to define nonlinear dynamics on a multi-agent network. We apply these frameworks to characterize a pitchfork bifurcation in a multi-agent network. For a graph with a single node, the proposed dynamics reduce to scalar nonlinear dynamics. In essence, the proposed dynamics are extensions of the scalar nonlinear dynamics to engineered multi-agent systems. The major contributions of our work are:

1) We propose generalized frameworks to describe distributed nonlinear dynamics in a multi-agent network.

2) For each framework, we generically define the set of final possible equilibrium configurations.

3) We define the distributed pitchfork bifurcation dynamics for networked systems using these frameworks.

4) We present some general tools to study stability of these dynamics, and utilize them to study stability of the pitchfork bifurcation dynamics.

5) We present a comprehensive treatment of these dynamics for lower order networks.

The remainder of the paper is organized as following. In the Section II, we elucidate some basics of dynamical systems and graph theory, which is followed by the development of frameworks to define nonlinear dynamics on graphs in Section III. We use these frameworks to study pitchfork bifurcation dynamics on graphs in Section IV. We further explain the results through some examples in Section V. Finally, our conclusions are in Section VI.

\section{PRELIMINARIES}

\section{A. Pitchfork bifurcation}

The equation

$$
\dot{x}=\gamma x-x^{3}, \quad \gamma, x \in \mathbb{R},
$$

is defined as the normal form for the supercritical pitchfork bifurcation [17]. The dynamics of (1) are as follows:

1) For $\gamma<0$, there exists a stable equilibrium point at $x=0$, and no other equilibrium point.

2) For $\gamma=0$, there exists a critically stable equilibrium point at $x=0$.

3) For $\gamma>0$, there exist two stable equilibrium points at $x= \pm \sqrt{\gamma}$, and an unstable equilibrium point at $x=0$.

The point $\gamma=0$ is called the bifurcation point. 


\section{B. Laplacian Matrix of a graph}

Given a digraph $\mathcal{G}=(V, \mathcal{E})$, where $V=\left\{v_{1}, \ldots, v_{n}\right\}$ is the set of nodes and $\mathcal{E}$ is the set of edges, the Laplacian matrix $\mathcal{L}(\mathcal{G}) \in \mathbb{R}^{n \times n}$ has entries:

$$
l_{i, j}= \begin{cases}-1, & \text { if }(i, j) \in \mathcal{E}, \\ d_{i}, & \text { if } i=j, \\ 0, & \text { otherwise }\end{cases}
$$

where $d_{i}$ is the out-degree of node $i$, i.e., number of edges emanating from node $i$ [3]. The set of nodes $j \in V$, such that $(i, j) \in \mathcal{E}$, is referred to as the adjacency of the node $i$, and is denoted $\operatorname{adj}(i)$.

Properties of Laplacian Matrix:

1) The Laplacian matrix is symmetric if and only if $\mathcal{G}$ is undirected.

2) A symmetric Laplacian matrix is positive semidefinite.

3) For a graph $\mathcal{G}$ with $n$ nodes and at least one globally reachable node, the rank of the Laplacian matrix is $n-1$.

4) The kernel of the Laplacian matrix for a graph $\mathcal{G}$ of order $n$ with at least one globally reachable node is $\operatorname{diag}\left(\mathbb{R}^{n}\right)$, i.e. $\left\{\left(x_{1}, \ldots, x_{n}\right) \in \mathbb{R}^{n} \mid x_{1}=\cdots=x_{n}\right\}$.

\section{Center manifold theorem}

For $\left(z_{1}, z_{2}\right) \in \mathbb{R}^{n_{1}} \times \mathbb{R}^{n_{2}}$, consider the following system

$$
\begin{aligned}
& \dot{z}_{1}=A_{1} z_{1}+g_{1}\left(z_{1}, z_{2}\right), \\
& \dot{z}_{2}=A_{2} z_{2}+g_{2}\left(z_{1}, z_{2}\right),
\end{aligned}
$$

where all eigenvalues of $A_{1} \in \mathbb{R}^{n_{1} \times n_{1}}$, and $A_{2} \in \mathbb{R}^{n_{2} \times n_{2}}$ have zero and negative real parts, respectively. The functions $g_{1}: \mathbb{R}^{n_{1}} \times \mathbb{R}^{n_{2}} \rightarrow \mathbb{R}^{n_{1}}$, and $g_{2}: \mathbb{R}^{n_{1}} \times \mathbb{R}^{n_{2}} \rightarrow \mathbb{R}^{n_{2}}$ satisfy the conditions

$$
g_{i}(0,0)=0, \quad \frac{\partial g_{i}}{\partial z}(0,0)=0, \quad \forall i \in\{1,2\} .
$$

For the system in equation (2), for small $z_{1}$, there exists [7] an invariant center manifold $h: \mathbb{R}^{n_{1}} \rightarrow \mathbb{R}^{n_{2}}$ satisfying the conditions

$$
\begin{gathered}
h(0)=0, \quad \frac{\partial h}{\partial z_{1}}(0)=0, \quad \text { and } \\
A_{2} h\left(z_{1}\right)+g_{2}\left(z_{1}, h\left(z_{1}\right)\right)=\frac{\partial h}{\partial z_{1}}\left(z_{1}\right)\left[A_{1} z_{1}+g_{1}\left(z_{1}, h\left(z_{1}\right)\right)\right] .
\end{gathered}
$$

The center manifold theorem [7] states that the dynamics on the center manifold determine the overall asymptotic dynamics of (2) near $\left(z_{1}, z_{2}\right)=(0,0)$, i.e., the overall dynamics are determined by

$$
\dot{z}_{1}=A_{1} z_{1}+g_{1}\left(z_{1}, h\left(z_{1}\right)\right) .
$$

\section{Laplacian flow}

Let $\mathcal{G}$ be a undirected connected graph of order $n$. The Laplacian flow on $\mathbb{R}^{n}$ is defined by

$$
\dot{x}=-\mathcal{L}(\mathcal{G}) x .
$$

In components, the Laplacian flow is given by

$$
\dot{x}_{i}=\sum_{j \in \operatorname{adj}(i)}\left(x_{j}-x_{i}\right), \quad i \in\{1, \ldots, n\} .
$$

The vector $\mathcal{L}(\mathcal{G}) x$ is called the disagreement vector. It has been shown in [13] that the solutions to the Laplacian flow converge to $\operatorname{diag}\left(\mathbb{R}^{n}\right)$ for fixed as well as switching topologies.

\section{DISTRIBUTED NONLINEAR DYNAMICS IN NETWORKS}

Before we define distributed nonlinear dynamics in networks, we introduce the following notation. We denote the set of connected undirected graphs with $n$ nodes by

$$
\Gamma_{n}=\left\{\mathcal{G} \mid \mathcal{L}(\mathcal{G})=\mathcal{L}(\mathcal{G})^{T} \text {, and } \operatorname{rank}(\mathcal{L}(\mathcal{G}))=n-1\right\} .
$$

\section{A. Absolute nonlinear flow}

We call a flow absolute nonlinear flow if each node transmits a value which is a function of only its own label. For a $\mathcal{G} \in \Gamma_{n}$, on $\mathbb{R}^{n}$, such a flow is given by

$$
\dot{x}=\mathcal{L}(\mathcal{G}) f(x),
$$

where $f: \mathbb{R}^{n} \rightarrow \mathbb{R}^{n}$ is a smooth function. In components, the absolute nonlinear flow is given by

$$
\dot{x}_{i}=\sum_{j \in \operatorname{adj}(i)}\left(f_{i}\left(x_{i}\right)-f_{j}\left(x_{j}\right)\right), \quad \forall i \in\{1, \ldots, n\} .
$$

The set of equilibrium points of the absolute nonlinear flow is

$$
\left\{x^{*} \mid f\left(x^{*}\right) \in \operatorname{diag}\left(\mathbb{R}^{n}\right)\right\} .
$$

The salient feature of the absolute nonlinear flow formulation is that the set of equilibrium points is an invariant over the set $\Gamma_{n}$. Moreover, the sum of the states is an invariant over any trajectory of the system, which follows from the fact that $\sum_{i=1}^{n} \dot{x}_{i}=0$.

\section{B. Disagreement nonlinear flow}

We call a flow disagreement nonlinear flow if each node transmits a value which is determined only by corresponding entry in the disagreement vector. For a $\mathcal{G} \in \Gamma_{n}$, on $\mathbb{R}^{n}$, such a flow is given by

$$
\dot{x}=f(\mathcal{L}(\mathcal{G}) x),
$$

where $f: \mathbb{R}^{n} \rightarrow \mathbb{R}^{n}$ is some smooth function. In components, the disagreement nonlinear flow is given by

$$
\dot{x}_{i}=f_{i}\left(\sum_{j \in \operatorname{adj}(i)}\left(x_{i}-x_{j}\right)\right), \quad \forall i \in\{1, \ldots, n\} .
$$

A particular case of the disagreement nonlinear flow is when each $f_{i}$ is a polynomial. In this scenario, the disagreement nonlinear flow is given by

$$
\dot{x}=\left(a_{0}+a_{1} \mathcal{D}(x)+\ldots+a_{m}(\mathcal{D}(x))^{m}\right) \mathbf{1}_{n},
$$

where $\mathcal{D}(x)=\operatorname{diag}(\mathcal{L}(\mathcal{G}) x)$. In components, this becomes $\dot{x}_{i}=a_{0}+a_{1} \mathcal{I}\left(x_{i}\right)+\ldots+a_{m}\left(\mathcal{I}\left(x_{i}\right)\right)^{m}, \quad \forall i \in\{1, \ldots, n\}$, where $\mathcal{I}\left(x_{i}\right)=\sum_{j \in \operatorname{adj}(i)}\left(x_{i}-x_{j}\right)$. Let the $r \leq m$ real roots of the equation

$$
a_{0}+a_{1} z+\ldots+a_{m} z^{m}=0
$$

be $z_{i}, i \in\{1, \ldots, r\}$. The set of equilibrium points of the disagreement nonlinear flow with polynomial nonlinearity is

$$
\left\{x^{*} \in \mathbb{R}^{n} \mid \mathcal{L}(\mathcal{G}) x^{*} \in\left\{z_{1}, \ldots, z_{r}\right\}^{n}\right\} .
$$

Here, the equilibrium points depend on the graph topology. 


\section{DISTRIBUTED BIFURCATIONS IN NETWORKS}

We study a particular class of distributed nonlinear dynamics where $f_{i}: \mathbb{R} \rightarrow \mathbb{R}$, for each $i \in\{1, \ldots, n\}$, is $f_{i}(x)=\gamma x-x^{3}$, where $\gamma \in \mathbb{R}$ is some constant. We refer to such nonlinearity as a pitchfork nonlinearity.

\section{A. Absolute nonlinear flow with pitchfork nonlinearity}

Given a connected undirected graph $\mathcal{G} \in \Gamma_{n}$, and $\gamma \in \mathbb{R}$, the absolute nonlinear flow with pitchfork nonlinearity is

$$
\dot{x}=\gamma \mathcal{L}(\mathcal{G}) x-\mathcal{L}(\mathcal{G}) \operatorname{diag}(x)^{3} \mathbf{1}_{n} .
$$

In components, this becomes

$$
\dot{x}_{i}=\gamma \sum_{j \in \operatorname{adj}(i)}\left(x_{i}-x_{j}\right)-\sum_{j \in \operatorname{adj}(i)}\left(x_{i}^{3}-x_{j}^{3}\right),
$$

for all $i \in\{1, \ldots, n\}$.

For a given graph $\mathcal{G} \in \Gamma_{n}$, and a full rank diagonal matrix $\Upsilon \in \mathbb{R}^{n \times n}$, let us define the generalized Laplacian flow by

$$
\dot{x}=-\mathcal{L}(\mathcal{G}) \Upsilon x .
$$

Lemma 1 (Generalized Laplacian Flow): For the generalized Laplacian flow, the following statements hold:

1) The equilibrium points are given by

$$
\mathcal{E}=\left\{\alpha \Upsilon^{-1} \mathbf{1}_{n} \mid \alpha \in \mathbb{R}\right\} .
$$

2) The solutions converge to the set $\mathcal{E}$ if and only if $\Upsilon>$ 0 .

Proof: The proof is similar to Exercise 1.25 in [3], with a Lyapunov function $V(x)=x^{T} \Upsilon \mathcal{L}(\mathcal{G}) \Upsilon x$. For the brevity, we omit the details.

Before we analyze the absolute nonlinear flow with pitchfork nonlinearity, we introduce some useful notation. Given $\gamma \in \mathbb{R}_{>0}$, define $f_{0}, f_{ \pm}:[-\sqrt{4 \gamma / 3}, \sqrt{4 \gamma / 3}] \rightarrow \mathbb{R}$ by

$$
f_{0}(\beta)=\beta \text {, and } f_{ \pm}(\beta)=-\frac{\beta}{2} \pm \sqrt{\gamma-\frac{3}{4} \beta^{2}} .
$$

Theorem 1 (Abs. nonlin. flow with pitchfork nonlinearity): For the absolute nonlinear flow with pitchfork nonlinearity, the following statements hold:

1) Equilibrium points:

For $\gamma \leq 0$, the set of equilibrium points is

$$
\mathcal{E}_{c}=\operatorname{diag}\left(\mathbb{R}^{n}\right) .
$$

For $\gamma>0$, the set of equilibrium points is

$$
\begin{array}{r}
\mathcal{E}_{b}=\left\{\left\{f_{-}(\beta), f_{0}(\beta), f_{+}(\beta)\right\}^{n} \mid\right. \\
\beta \in[-\sqrt{4 \gamma / 3}, \sqrt{4 \gamma / 3}]\},
\end{array}
$$

where $\left\{f_{-}(\beta), f_{0}(\beta), f_{+}(\beta)\right\}^{n}$ is the set of $n$-tuples which have each entry in the set $\left\{f_{-}(\beta), f_{0}(\beta), f_{+}(\beta)\right\}$.

2) Consensus:

For $\gamma \leq 0$, each trajectory converges to some point in the set $\mathcal{E}_{c}$.

3) Bifurcation:

For $\gamma>0$, each equilibrium point $x^{*} \in \mathcal{E}_{b}$ is locally stable if and only if $3 x_{i}^{* 2}>\gamma$ for each $i \in\{1, \ldots, n\}$.
Proof: We start by determining the equilibrium points for equation (5), which are given by

$$
\begin{aligned}
& \gamma x-\operatorname{diag}(x)^{3} \mathbf{1}_{n} \in \operatorname{ker}(\mathcal{L}(\mathcal{G})), \\
\Longrightarrow & \gamma x_{i}-x_{i}^{3}=\alpha, \quad \forall i \in\{1, \ldots, n\}, \text { and } \alpha \in \mathbb{R} .
\end{aligned}
$$

We observe that equation (9) is a cubic equation and hence, has at least one real root $\beta$ (say). The other roots of the equation (9) can be determined in terms of $\beta$, and are given by

$$
x_{i}=-\frac{\beta}{2} \pm \sqrt{\gamma-\frac{3}{4} \beta^{2}}, \quad \forall i \in\{1, \ldots, n\} .
$$

We observe that the roots given in equation (10) are complex if $\gamma \leq 0$. Hence, for $\gamma \leq 0$, the equilibrium points are given by the set $\mathcal{E}_{c}$. It follows from equation (10) that for $\gamma>0$, $\mathcal{E}_{b}$ is the set of equilibrium points.

To establish the second statement, we consider a Lyapunov function $V(x)=x^{T} \mathcal{L}(\mathcal{G}) x$. We observe that, for $\gamma \leq 0$, the Lie derivative of this Lyapunov function along the absolute nonlinear flow with pitchfork nonlinearity is given by

$$
\dot{V}(x)=2 \gamma x^{T} \mathcal{L}(\mathcal{G}) x-2 x^{T} \mathcal{L}(\mathcal{G}) \operatorname{diag}(x)^{3} \mathbf{1}_{n} \leq 0,
$$

which establishes the stability of each point in the set $\mathcal{E}_{c}$. The proof of convergence is similar to Exercise 1.25 in [3].

To establish the third statement, we linearize the absolute nonlinear flow with pitchfork nonlinearity about an equilibrium point $x^{*}$ to get

$$
\dot{x}=\mathcal{L}(\mathcal{G})\left(\gamma I-3 \operatorname{diag}\left(x^{*}\right)^{2}\right) x=: \mathcal{L}(\mathcal{G}) \Upsilon x,
$$

where $\Upsilon$ is a diagonal matrix. From Lemma 1, it follows that each equilibrium point $x^{*} \in \mathcal{E}_{b}$ is locally stable if and only if $\Upsilon$ is negative definite, which concludes the proof.

Remark 1: Let $\Xi$ be the set of $n$-dimensional vectors with entries in $\{-, 0,+\}$, whose cardinality is $3^{n}$. Therefore, $\xi \in$ $\Xi$ is an $n$-dimensional multi-index with indices in alphabet $\{-, 0,+\}$. For any $\xi \in \Xi$, define $f_{\xi}:[-\sqrt{4 \gamma / 3}, \sqrt{4 \gamma / 3}] \rightarrow$ $\mathbb{R}^{n}$ by

$$
f_{\xi}(\beta)=\left(f_{\xi_{1}}(\beta), \ldots, f_{\xi_{n}}(\beta)\right) \in \mathbb{R}^{n} .
$$

The set $\mathcal{E}_{b}$ can be interpreted as the union of three curves in the following way

$$
\mathcal{E}_{b}=\cup_{\xi \in \Xi} f_{\xi}([-\sqrt{4 \gamma / 3}, \sqrt{4 \gamma / 3}]) .
$$

(Here we let $g(A)$ denote the image of a function $g: A \rightarrow$ R.)

Remark 2: The results in Theorem 1 hold for any directed graph with at least one globally reachable node.

Conjecture 1 (Completeness): Given a $\gamma \in \mathbb{R}$, the union of the basin of attractions of all the stable equilibrium points of the absolute nonlinear flow with pitchfork nonlinearity is $\mathbb{R}^{n} \backslash \mathcal{Z}$, where $\mathcal{Z}$ is a measure zero set.

Conjecture 2 (Switching topology): The results in Theorem 1 hold for a network with switching topology $\mathcal{G}_{k} \in$ $\Gamma_{n}, k \in \mathbb{N}$. 


\section{B. Disagreement nonlinear flow with pitchfork nonlinearity}

Given a connected undirected graph $\mathcal{G} \in \Gamma_{n}$, and $\gamma \in \mathbb{R}$, the disagreement nonlinear flow with pitchfork nonlinearity is

$$
\dot{x}=\gamma \mathcal{L}(\mathcal{G}) x-(\operatorname{diag}(\mathcal{L}(\mathcal{G}) x))^{3} \mathbf{1}_{n} .
$$

In components, the above dynamics, $\forall i \in\{1, \ldots, n\}$, are given by

$$
\dot{x_{i}}=\gamma \sum_{j \in \operatorname{adj}(i)}\left(x_{i}-x_{j}\right)-\left(\sum_{j \in \operatorname{adj}(i)}\left(x_{i}-x_{j}\right)\right)^{3} .
$$

Before we analyze the disagreement nonlinear flow with pitchfork nonlinearity, we introduce the following notation. We partition the Laplacian matrix in the following way:

$$
\mathcal{L}(\mathcal{G})=\left[\begin{array}{cc}
L_{n-1} & L_{*, n} \\
L_{n, *} & L_{n, n}
\end{array}\right],
$$

where $L_{n-1} \in \mathbb{R}^{(n-1) \times(n-1)}$.

We also construct a transformation matrix $P \in \mathbb{R}^{n \times n}$ in the following way:

$$
P=\left[\begin{array}{cc}
L_{n-1} & L_{*, n} \\
\mathbf{1}_{n-1}^{T} & 1
\end{array}\right] .
$$

The last row of the transformation matrix $P$ is chosen to be the basis of the kernel of the Laplacian matrix $\mathcal{L}(\mathcal{G})$, for $\mathcal{G} \in \Gamma_{n}$. Hence, a coordinate transform through matrix $P$ separates the center manifold and the stable/unstable manifold. Now, we state some properties of the transformation matrix $P$.

Lemma 2 (Properties of the transformation matrix): Given a graph $\mathcal{G} \in \Gamma_{n}$, then for the transformation matrix $P$ defined in equation (14) the following statements hold:

1) The submatrix $L_{n-1}$ is symmetric positive definite.

2) The transformation matrix $P$ is full rank.

3) The inverse of the transformation matrix satisfies the following:

$$
\mathbf{1}_{n}^{T} P^{-1}=e_{n}^{T}, \quad \text { and } \quad P^{-1} e_{n}=\frac{1}{n} \mathbf{1}_{n},
$$

where $e_{n}=\left[\begin{array}{llll}0 & \ldots & 0 & 1\end{array}\right]^{T}$.

Proof: For the brevity, we present only the idea of the proof. The first statement follows from some algebraic manipulations on the Laplacian matrix, Theorem 1.37 in [3], and semi-positive definiteness of the Laplacian matrix. The second statement follows from the first statement and the fact that $\mathbf{1}_{n}$ belongs to the kernel of the Laplacian matrix.

To prove the third statement, we note that the inverse of transformation matrix $P$ is given by

$$
P^{-1}=\left[\begin{array}{cc}
\left(L_{n-1}-L_{*, n} \mathbf{1}_{n-1}^{T}\right)^{-1} & \frac{1}{n} \mathbf{1}_{n-1} \\
-\mathbf{1}_{n-1}^{T}\left(L_{n-1}-L_{*, n} \mathbf{1}_{n-1}^{T}\right)^{-1} & \frac{1}{n}
\end{array}\right] .
$$

It follows immediately from equation (15) that $\mathbf{1}_{n}^{T} P^{-1}=e_{n}$ and $P^{-1} e_{n}=\frac{1}{n} \mathbf{1}_{n}$. This concludes the proof of the third and the last statement.

Theorem 2 (Dis. nonlin. flow with pitchfork nonlinearity): For the disagreement nonlinear flow with pitchfork nonlinearity, the following statements hold:

\section{Equilibrium points:}

1) For $\gamma \leq 0$, the set of equilibrium points is

$$
\mathcal{F}_{c}=\operatorname{diag}\left(\mathbb{R}^{n}\right) .
$$

2) For $\gamma>0$, the set of equilibrium points is

$$
\begin{aligned}
\mathcal{F}_{b}=\left\{P^{-1} y \mid y\right. & \in\{0,-\sqrt{\gamma}, \sqrt{\gamma}\}^{n-1} \times \mathbb{R}, \\
& \text { and } \left.\sum_{i=1}^{n-1} y_{i} \in\{0,-\sqrt{\gamma}, \sqrt{\gamma}\}\right\} .
\end{aligned}
$$

\section{Consensus:}

For $\gamma \leq 0$, each trajectory converges to some point in the set $\mathcal{F}_{c}$.

\section{Bifurcation:}

1) For $\gamma>0$, and $n$ even, the set of locally stable equilibrium points is

$$
\begin{aligned}
\overline{\mathcal{F}}_{b}=\left\{P^{-1} y \mid y \in\right. & \{-\sqrt{\gamma}, \sqrt{\gamma}\}^{n-1} \times \mathbb{R}, \\
& \text { and } \left.\sum_{i=1}^{n-1} y_{i} \in\{-\sqrt{\gamma}, \sqrt{\gamma}\}\right\} .
\end{aligned}
$$

Moreover, each equilibrium point $x^{*} \in \mathcal{F}_{b} \backslash \overline{\mathcal{F}}_{b}$ is unstable.

2) For $\gamma>0$, and odd $n>1$, each equilibrium point $x^{*} \in \mathcal{F}_{b}$ is unstable.

Proof: We transform the coordinates to $y=P x$, and observe that in the new coordinates the equation (11) transforms to

$$
P^{-1} \dot{y}=\gamma\left[\begin{array}{c}
y_{1}-y_{1}^{3} \\
\vdots \\
y_{n-1}-y_{n-1}^{3} \\
-\sum_{i=1}^{n-1} y_{i}+\left(\sum_{i=1}^{n-1} y_{i}\right)^{3}
\end{array}\right] .
$$

With some algebraic manipulations, one may see that the system in equation (16) is equivalent to

$$
\begin{gathered}
{\left[\begin{array}{c}
\dot{y}_{1} \\
\vdots \\
\dot{y}_{n-1}
\end{array}\right]=\gamma\left(L_{n-1}-L_{*, n} \mathbf{1}_{n-1}^{T}\right)\left[\begin{array}{c}
y_{1}-y_{1}^{3} \\
\vdots \\
y_{n-1}-y_{n-1}^{3} \\
+L_{*, n} \dot{y}_{n}
\end{array}\right]} \\
\text { and } \dot{y}_{n}=-\sum_{i=1}^{n-1} y_{i}^{3}+\left(\sum_{i=1}^{n-1} y_{i}\right)^{3}
\end{gathered}
$$

To establish the first statement, we note that the equilibrium point of the system in equation (17), for each $i \in$ $\{1, \ldots, n-1\}$, are given by

$$
y_{i}^{*} \in \begin{cases}\{0\}, & \text { if } \quad \gamma \leq 0, \\ \{0, \pm \sqrt{\gamma}\} & \text { if } \quad \gamma>0 .\end{cases}
$$

The equilibrium points, thus obtained, should be consistent with the equilibrium condition of equation (18). Substitution of these equilibrium points into the equation (18) yields $\sum_{i=1}^{n-1} y_{i} \in\{0, \pm \sqrt{\gamma}\}$. The equilibrium value of $y_{n}$ is a free 
parameter, and can take any value $\beta \in \mathbb{R}$. This concludes the proof of the first statement.

The proof of the second statement is similar to the Lyapunov function based proof in Theorem 1.

To prove the local stability of each equilibrium point $x^{*} \in$ $\overline{\mathcal{F}}_{b}$, for $n$ even, we shift the origin of (17) and (18), defining new coordinates as

$$
\left(\zeta_{1}, \zeta_{2}\right)^{T}=\left(\zeta_{1_{1}}, \ldots, \zeta_{1_{n-1}}, \zeta_{2}\right)^{T}=y-y^{*},
$$

where $P^{-1} y^{*} \in \overline{\mathcal{F}}_{b}$. In these new coordinates, (17) and (18) become

$$
\begin{array}{r}
{\left[\begin{array}{c}
\dot{\zeta}_{1} \\
\dot{\zeta}_{2}
\end{array}\right]=\left[\begin{array}{rr}
-2 \gamma L_{n-1}\left(I+\mathbf{1}_{n-1} \mathbf{1}_{n-1}^{T}\right) & 0 \\
0 & 0
\end{array}\right]} \\
\times\left[\begin{array}{c}
\zeta_{1} \\
\zeta_{2}
\end{array}\right]+\left[\begin{array}{c}
\bar{g}_{1}\left(\zeta_{1}\right) \\
\bar{g}_{2}\left(\zeta_{2}\right)
\end{array}\right],
\end{array}
$$

where $\bar{g}_{1}: \mathbb{R}^{n-1} \rightarrow \mathbb{R}^{n-1}$ and $\bar{g}_{2}: \mathbb{R}^{n-1} \rightarrow \mathbb{R}$ satisfy equation (3).

The dynamics of (19) are similar to the dynamics of (2), and $\zeta_{1}=h\left(\zeta_{2}\right)=0$ is the center manifold. The $\zeta_{2}$ dynamics on this manifold are neutrally stable. Hence, each equilibrium point $x^{*} \in \overline{\mathcal{F}}_{b}$ is locally stable.

Similarly, for $n$ odd, expressing (17) and (18) in the new coordinates gives

$$
\begin{array}{r}
{\left[\begin{array}{c}
\dot{\zeta}_{1} \\
\dot{\zeta}_{2}
\end{array}\right]=\left[\begin{array}{rr}
\gamma L_{n-1}\left(-2 I+\mathbf{1}_{n-1} \mathbf{1}_{n-1}^{T}\right) & 0 \\
-3 \gamma \mathbf{1}_{n-1}^{T} & 0
\end{array}\right]} \\
\times\left[\begin{array}{c}
\zeta_{1} \\
\zeta_{2}
\end{array}\right]+\left[\begin{array}{c}
g_{1}\left(\zeta_{1}\right) \\
g_{2}\left(\zeta_{2}\right)
\end{array}\right],
\end{array}
$$

where, $g_{1}: \mathbb{R}^{n-1} \rightarrow \mathbb{R}^{n-1}$, and $g_{2}: \mathbb{R}^{n-1} \rightarrow \mathbb{R}$ satisfy the conditions in equation (3). Since, the matrix $-2 I+$ $\mathbf{1}_{n-1} \mathbf{1}_{n-1}^{T}$ has an eigenvalue at $n-3$, the equilibria are unstable for $n \geq 3$.

The instability of the set $\mathcal{F}_{b} \backslash \overline{\mathcal{F}}_{b}$ follows similarly.

Remark 3: The absolute and disagreement nonlinear flows can be studied with other normal forms for the bifurcations in scalar systems. For example, one may consider the transcritical nonlinearity $f_{i}: \mathbb{R} \rightarrow \mathbb{R}$ defined by $f_{i}(x)=\gamma x-x^{2}$, for all $i \in\{1, \ldots, n\}$, and some $\gamma \in \mathbb{R}$. It can be shown that, for $\gamma>0$, the absolute nonlinear flow with transcritical nonlinearity converges to consensus under very restrictive conditions, otherwise each equilibrium point is unstable. The disagreement nonlinear flow with transcritical nonlinearity has each equilibrium point unstable for $\gamma>0$.

\section{NUMERICAL RESUltS}

We determined the equilibrium points of the absolute nonlinear flow with pitchfork nonlinearity and established their stability in Theorem 1. Now we study this system on some lower order graphs to better understand the underlying dynamics. We start with a graph with two nodes. For $\gamma \leq 0$, the set of equilibrium points of this system is the consensus set, $\operatorname{diag}\left(\mathbb{R}^{2}\right)$, which are all stable, while for $\gamma>0$, the set of equilibrium points is shown in Figure 1(a). The subset of the consensus set $\mathcal{C}_{2}$ belonging to the convex hull of the set $\mathcal{E}_{2}$ is unstable. As $\gamma$ is decreased, the ellipse of equilibrium points shrinks in size, disappearing at $\gamma=0$. Observe that $x_{1}+x_{2}$ is an invariant along any trajectory of the system, and it can be utilized to reduce the dimension of the system. For the reduced system $x_{1}+x_{2} \equiv c$ is a parameter, and it turns out that a pitchfork bifurcation is observed at $c=\sqrt{4 \gamma / 3}$. The corresponding bifurcation diagram for $\gamma=1$ is shown in Figure $1(\mathrm{~b})$. For $c \geq \sqrt{4 \gamma / 3}$, the only equilibrium point of the system is at $x=c / 2$. For $c<\sqrt{4 \gamma / 3}$, this equilibrium point loses its stability and two new stable equilibrium points appear in the system. This is a pitchfork bifurcation.

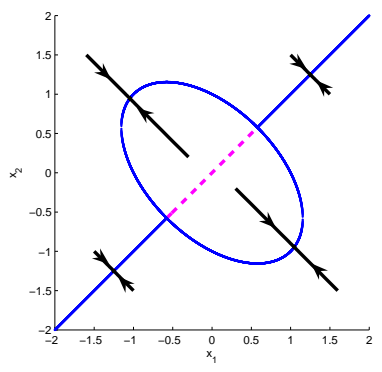

(a) Equilibrium points

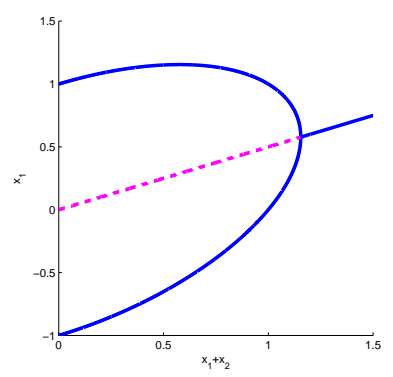

(b) Bifurcation diagram

Fig. 1. Absolute nonlinear flow with pitchfork nonlinearity on a graph with two nodes and $\gamma=1$. (a) The unstable equilibrium points are shown with magenta color while the stable ones are shown in blue color. (b) The bifurcation diagram for the reduced system. Notice the pitchfork bifurcation at $x_{1}+x_{2}=2 / \sqrt{3}$.

We now consider a line graph with three nodes. For $\gamma \leq 0$, the set of equilibrium points is the consensus set, $\operatorname{diag}\left(\mathbb{R}^{3}\right)$, which are all stable. The set of equilibrium points for $\gamma=$ 1 is shown in Figure 2(a). Similar to the two node case, $x_{1}+x_{2}+x_{3}$ is an invariant along any trajectory of the system, and this can be utilized to reduce the dimension of the system. For the reduced system $x_{1}+x_{2}+x_{3} \equiv c$ is a parameter, and very interesting behaviors are observed as this parameter is varied (see Figure 2(b)). We note that the equilibrium at $(c / 3, c / 3)$ corresponds to the consensus state. For $c=0$ the set of equilibrium points is the consensus point and an ellipse. Each point on the ellipse is stable, while the consensus point is a source. As the value of $c$ is increased from zero, the reduced system has seven equilibrium points, three of which are sinks, three are saddle points, and one is a source. As the value of $c$ is further increased the three saddle points move towards the source, reaching it at $c=\sqrt{3 \gamma}$ at an $S_{3}$-symmetric transcritical bifurcation [2], [8]. As the saddle points cross the source, i.e., for $c>\sqrt{3 \gamma}$, the source becomes a sink, and the three saddle points move towards the other 
three sinks. At $c=2 \sqrt{\gamma}$, the three saddles meet the three sinks and annihilate each other in saddlenode bifurcations. For $c>2$, there is only one equilibrium point in the system, which is a sink.

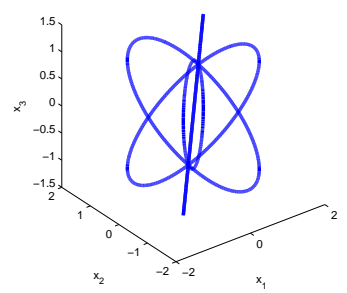

(a) Equilibrium points

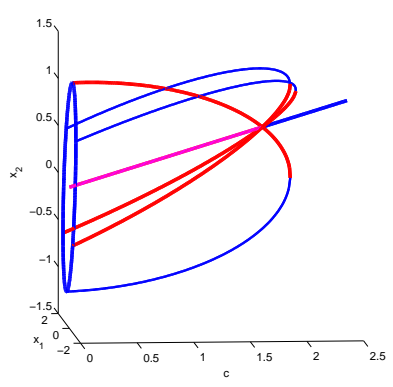

(b) Bifurcation diagram

Fig. 2. Absolute nonlinear flow with pitchfork nonlinearity on a line graph with three nodes and $\gamma=1$. (a) The equilibrium points are comprised of three ellipses and a line. (b) The bifurcation diagram for the reduced system. Notice the $S_{3}$-symmetric transcritical bifurcation at $c=\sqrt{3}$, and the saddlenode bifurcations at $c=2$.

We now study the disagreement nonlinear flow with pitchfork nonlinearity on a line graph with two nodes. For $\gamma \leq 0$, the set of equilibrium points for this system is the consensus set, $\operatorname{diag}\left(\mathbb{R}^{2}\right)$, and each equilibrium point is stable. For $\gamma=1$, the set of equilibrium points is shown in Figure 3. For $\gamma>0$ each point in the consensus set is unstable, while all other equilibrium points are stable.

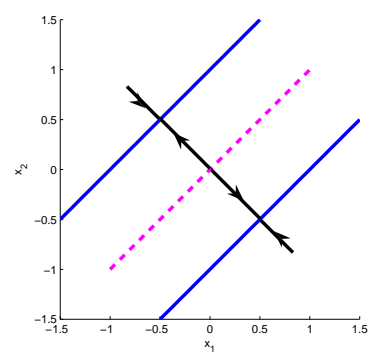

Fig. 3. Phase plot for relative nonlinear flow with pitchfork nonlinearity on a graph with two nodes and $\gamma=1$. The consensus set (shown in magenta) is unstable, while two sets (shown in blue) are stable.

\section{CONCLUSIONS}

In this paper, we considered three frameworks which define distributed nonlinear dynamics in multi-agent networks. We determined the set of equilibria that could be achieved through these dynamics, and examined their stability. We also described the bifurcation behavior in multi-agent networks using these frameworks, and demonstrated a variety of interesting behaviors that can be achieved.

A number of extensions to the work presented here are possible. For example, the networks considered here are static. There is a high possibility that the described dynamics persist for networks with switching topology as well. Furthermore, the class of functions which yield stable equilibria is not well understood yet. It remains an open problem to characterize this.

\section{REFERENCES}

[1] M. Arcak. Passivity as a design tool for group coordination. IEEE Transactions on Automatic Control, 52(8):1380-1390, 2007.

[2] P. Ashwin, O. Burylko, and Y. Maistrenko. Bifurcation to heteroclinic cycles and sensitivity in three and four coupled phase oscillators. Physica D: Nonlinear Phenomena, 237(4):454-466, 2008.

[3] F. Bullo, J. Cortés, and S. Martínez. Distributed Control of Robotic Networks. Applied Mathematics Series. Princeton University Press, 2009. Available at http://www.coordinationbook.info.

[4] I. D. Couzin, J. Krause, N. R. Franks, and S. A. Levin. Effective leadership and decision-making in animal groups on the move. Nature, 433(7025):513-516, 2005.

[5] J. A. Fax and R. M. Murray. Information flow and cooperative control of vehicle formations. IEEE Transactions on Automatic Control, 49(9):1465-1476, 2004.

[6] A. Jadbabaie, J. Lin, and A. S. Morse. Coordination of groups of mobile autonomous agents using nearest neighbor rules. IEEE Transactions on Automatic Control, 48(6):988-1001, 2003.

[7] H. K. Khalil. Nonlinear Systems. Prentice Hall, 3 edition, 2002.

[8] M. Kimura and J. Moehlis. Novel vehicular trajectories for collective motion from coupled oscillator steering control. SIAM Journal on Applied Dynamical Systems, 7(4):1191-1212, 2008

[9] H. Kwatny, A. Pasrija, and L. Bahar. Static bifurcations in electric power networks: Loss of steady-state stability and voltage collapse. IEEE Transactions on Circuits and Systems, 33(10):981-991, 1986.

[10] J. Lin, A. S. Morse, and B. D. O. Anderson. The multi-agent rendezvous problem. Part 1: The synchronous case. SIAM Journal on Control and Optimization, 46(6):2096-2119, 2007.

[11] J. Lorenz. Continuous opinion dynamics under bounded confidence: a survey. International Journal of Modern Physics C, 18(12):18191838, 2007.

[12] R. Olfati-Saber, J. A. Fax, and R. M. Murray. Consensus and cooperation in networked multi-agent systems. Proceedings of the IEEE, 95(1):215-233, 2007.

[13] R. Olfati-Saber and R. M. Murray. Consensus problems in networks of agents with switching topology and time-delays. IEEE Transactions on Automatic Control, 49(9):1520-1533, 2004.

[14] L. Olien and J. Bélair. Bifurcations, stability, and monotonicity properties of a delayed neural network model. Physica D: Nonlinear Phenomena, 102(3-4):349-363, 1997.

[15] A. Papachristodoulou and A. Jadbabaie. Synchronization in oscillator networks with heterogeneous delays, switching topologies and nonlinear dynamics. In IEEE Conf. on Decision and Control, pages 4307-4312, San Diego, CA, December 2006.

[16] D. P. Spanos, R. Olfati-Saber, and R. M. Murray. Approximate distributed Kalman filtering in sensor networks with quantifiable performance. In Symposium on Information Processing of Sensor Networks, pages 133-139, Los Angeles, CA, April 2005.

[17] S. H. Strogatz. Nonlinear Dynamics and Chaos: With Applications to Physics, Biology, Chemistry, and Engineering. Perseus Books Group, 2000.

[18] H. G. Tanner, A. Jadbabaie, and G. J. Pappas. Flocking in fixed and switching networks. IEEE Transactions on Automatic Control, 52(5):863-868, 2007.

[19] J. Wei and S. Ruan. Stability and bifurcation in a neural network model with two delays. Physica D: Nonlinear Phenomena, 130(34):255-272, 1999.

[20] F. Zou and J. A. Nossek. Bifurcation and chaos in cellular neural networks. IEEE Transactions on Circuits and Systems I: Fundamental Theory and Applications, 40(3):166-173, 1993. 